\section{TWO CENTURIES AFTER DISCOVERY OF SELENIUM, STILL BETWEEN MEDICAL USE AND MISUSE IN CLINICAL PRACTICE?}

Selenium (Se), atomic number 34, was discovered as a by-product of sulfuric acid in 1817 by the Swedish chemist and physician J.J.

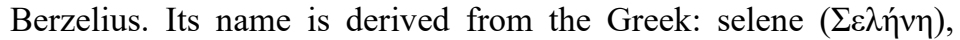
meaning Moon [1]. In Greek mythology, Selene is the goddess of the Moon. Namely when Se cools rapidly on being melted, it produces sheen similar to that of the Moon [2]. Appropriately, Se was detected in the Moon dust brought by the 1969 Apollo mission [3].

Although discovered before more than two centuries, Se and its role in health and disease remained underestimated and not fully elucidated. Endemic deficiency in large areas of central Asia clearly demonstrated its critical importance for human health and survival. There are two well-established entities of selenopenia: Kashin-Beck osteochondropathy and Keshan disease, severe cardiomyopathy characterized by fulminant heart failure [4, 5]. Concomitant with severe iodine deficiency, selenopenia leads to myxedematous endemic cretinism [6]. Se is present in food in organic form (selenocysteine and selenomethionine) and inorganic form (selenite or selenate). Recommended daily Se intake is 40-70 $\mu \mathrm{g}$. Plasma Se levels should be kept between 60 and $120 \mu \mathrm{g} / \mathrm{l}$ [7]. It is an active component of selenoproteome, consisted of about 26 selenoproteins, mainly enzymes. The most important are glutathione peroxidases (GPxs; encoded by seven genes), iodothyronine deiodinases (DIs; encoded by three genes,) and three isoforms of thioredoxin reductases (TRx). GPxs protect cells from oxidative stress, the TRx system is involved in development and proliferation. Deiodinase system is essential for T4 to T3 conversion and preservation of iodine from uncontrollable wasting [2, 7]. Underactivity of selenoproteins (especially GPxs) caused by Se deficiency increases the risk for the impaired outcome of pregnancy, exaggerated inflammation, autoimmunity, infertility, cancer, and osteochondropathy $[1,2,4,8]$. On the other hand, higher concentrations could potentiate the risk of hyperglycemia, insulin resistance, T2DM (Type 2 diabetes mellitus), glaucoma, cancer, hyperlipidemia, atherosclerosis, and increased cardiovascular mortality [2,9].

Se antiviral effects are nowadays of special interest, as well as its immunomodulatory properties. Namely, Se's ability to improve the activity of $\mathrm{T}$ cells and the cytotoxicity of natural killer cells could render it effective in viral diseases [2]. Se deficiency as an environmental factor increases T-cell activation and shifts Th1/Th2 ratio to a Th1-type response, increasing typical Th1 cytokines as IL-2, TNF- $\alpha$, IFN- $\gamma$, thereby reducing CD25+T regulatory cells (TREG) $[1,2,4,9]$. It is an immune response typical for autoimmune diseases. However, a recent meta-analysis did not present sufficient data on the clinical efficacy of selenium supplementation in chronic autoimmune thyroiditis (CAT) even in adults, and the authors conclud-

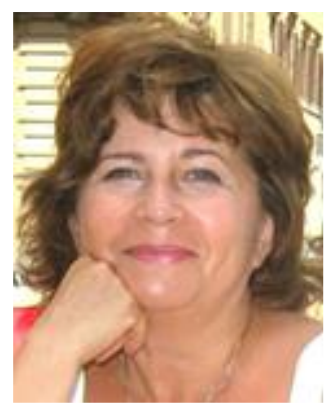

Correspondence to: Ljiljana Šaranac, M.D., Ph.D.

Faculty of Medicine, Dr. Zoran Djindjić 81 Blvd., Niš, Serbia

Phone: +381628242161

E-mail: endoljilja@yahoo.com

Received February $10^{\text {th }}, 2021$ 
ed that further investigations are warranted [10]. Interventional studies with Se in different pathological conditions in children and adolescents are very rare. Investigation of Se levels in critical illness and its supplementation is of special clinical interest. The use of systematic reviews (SR) as a reliable source of evidence for making health decisions is increasing rapidly. The systematic review is the objective and reproducible method that uses explicit strategies to systematically search all available evidence on a specific focused topic, critically appraise methods and results of these studies, and synthesize their findings in order to provide reliable and accurate conclusions [11]. Depending on the heterogeneity between the included studies, the SR may or may not provide a quantitative synthesis of the overall effect using data presented in each included primary study through a statistical method called meta-analysis [12, 13]. Evidence-based medicine uses a hierarchy of reliable evidence, and systematic reviews and meta-analysis are placed at the top of the Evidence-Based Pyramid, representing the strongest and highest quality of evidence available. Going down the pyramid, the quality of provided scientific evidence is decreasing. When synthesizing the results of the primary studies, SR uses efficient strategies to reduce bias and random errors. Reading the SR allows taking into account a different range of findings from multiple studies on a specific topic and an efficient way to keep up-to-date with the best available evidence. It is often used as a starting point when making clinical guidelines. This approach can also point out where the evidence is lacking and can direct future research efforts to fill these gaps. In this issue of Facta Universitatis Series Medicine and Biology, Vuković et al. presented results of their SR on the investigation of Se levels in endocrine diseases in childhood and concluded that level of Se was mostly lower in children with goiter and those with the T1DM while several significant correlations, positive and negative, were reported across different parameters in patients with thyroid disease and with diabetes. They found that goiter and thyroid dysfunction are more prominent signs of Se deficiency in school children, based mainly on studies performed in Iran and Turkey. This is not surprising taking into account that the human thyroid represents the organ with the highest Se concentration per unit weight, among all tissues. The thyroid retains its Se content to an even higher level than the brain does [1,7].

It is noteworthy to emphasize that selenopenia in obese children is responsible for impaired thyroid structure and function that could be misdiagnosed as CAT. Improving Se status could be crucial for avoiding thyroid dysfunction. Elevation of TSH level in obese children may be due to increased leptin-mediated production of pro$\mathrm{TRH}$, impaired feedback due to a lowered number of $\mathrm{T} 3$ receptors in the hypothalamus of obese subjects, or with great certainty because of variations in peripheral deiodinase activity due to relative Se deficiency in obese children [14]. Nevertheless, we still recommend Se use without estimation of the patient's Se status or precise measurements of its plasma concentrations. Doctors are prone to prescribe Se to every single patient with thyroid disease, neglecting or not be fully aware of its potentially harmful effects. Despite numerous favorable functions in health protection, the narrow range of Se safety should be a reason for its limited use. Namely, both acute and chronic Se toxic effects (selenosis) are possible [2, 7]. The high amount of inorganic Se in drinking water increases the relative risk for amyotrophic lateral sclerosis [15].

In an extensive review, Duntas and Benvenga showed dose dependent, beneficial and harmful effects of Se and the importance 
of selenostasis for maintenance of overall health [2]. The Se curve of plasma concentration is $\mathrm{U}$ shaped. The right amount of Se is necessary for beneficial effects and the proper function of the human body machinery. As for iodine too little and too high concentration could be harmful. Taking into account its effect on potentiation the antioxidant effect, the question should arise: "Does everyone need an antioxidant activity?". Obviously, there is no universal fit. A better way is to have an individual approach, and the best way is to consider Se use in different pathological conditions on the basis of Se level determination. Endocrinologists embraced Se use in autoimmune thyroid disease (AITD), but there is a critical appraisal for such use. Se use in incipient and mild forms of thyroid disease is beneficial and routine use in all patients with AITD is questioned. There is evidence of a potential effect of Se in reducing the TPO-antibodies (Abs) titer and improving the ultrasound appearance and structure. Optimal results were found in patients with low Se levels and those with very high TPO-Abs levels $[16,17]$.

Is the Se supplementation a simple solution for complicated health issues? This question still needs an answer. Se as a missing link between health and prevention of different diseases certainly merits further investigation. Recent information from the USA regarding centenarian who survived deadly disease is in favor of such opinion. In 1918, Bernice Homan was just a year old when the Spanish Flu swept across the world and nearly took her life. The doctor told her mother Bernice wouldn't survive the night. Despite a bad prognosis, her mother refused to give up. She heard that pumpkin seeds were the most helpful for the flu. She made Pumpkin Seed Tea and spoon-fed Bernice the whole night until the sun came up and Bernice opened her eyes [18]. Mrs. Homan is a living breathing miracle and this touching story has a connection with the fact that Se could be critical for survival. Namely pumpkin seed contains iodine with antibacterial and Se with antiviral effects [19]. Over 100 years later, Bernice was the first resident at Willamette Oaks in the USA, to roll up her sleeves and get the COVID-19 vaccine.

\section{References}

1. Duntas LH. Selenium and the thyroid: a close-knit connection. J Clin Endocrinol Metab 2010; 95 (12): 5180-5188, doi: 10.1210/jc.2010-0191

2. Duntas LH, Benvenga S. Selenium: an element for life. Endocrine 2015; 48:756-775.

3. Flohe L, Andreesen JR, Brigelius-Flohe R, Maiorino M, Ursini F. Selenium, the element of the moon, in the life on the earth. IUBMB Life 2000; 49:411-420.

4. Guastamacchia E, Giagulli VA, Licchelli B, Triggiani V. Selenium and Iodine in autoimmune thyroiditis. Endocrine, Metabolic\&Immune Disorders-Drug Targets 2015; 15:288-292.

5. Chanoine JP, Neve J, Wu S, Vanderpas J, Bourdoux P. Selenium decreases thyroglobulin concentrations but does not affect the increased thyroxine-totriiodothyronine ratio in children with congenital hypothyroidism. J Clin Endocrinol Metab 2010; 96:1160-1163.

6. Zimmermann MB, Kohrle J. The impact of of iron and selenium deficiencies on iodine and thyroid metabolism: biochemistry and relevance to public health. Thyroid 2002; 12:867-878.

7. Ashton K, Hooper L, Harvey LJ, Hurst R, Casgrain A, Fairweather-Tait SJ Methods of assessment of selenium status in humans: a systematic review. Am J Clin Nutr 2009; 89(Suppl):2025-2039.

8. Hofstee P, McAlpine JJ, McKeating DR, Vanderlelie JJ , Cuffe JSM, V Perkins AV. Low serum selenium in pregnancy is associated with reduced T3 and increased risk of GDM. J Endocrinol 2021; 248 (1):45-57. DOI: https://doi.org/10.1530/JOE-20-0319

9. Duntas LH. The role of iodine and selenium in autoimmune Thyroiditis Horm Metab Res 2015; 47:721-726.

10. Winther KH, Wichman JEM, Bonnema SJ, Hegedus L. Insufficient documentation for clinical efficacy of selenium supplementation in chronic 
autoimmune thyroiditis, based on a systematic review and meta-analysis. Endocrine 2017; 55:376-385

11. Gopalakrishnan S, Ganeshkumar P, Systematic Reviews and Meta-analysis: Understanding the Best Evidence in Primary Healthcare. J Family Med Prim Care 2013; 2(1):9-14. doi: 10.4103/2249-4863.109934.

12. McNamara ER, Scales CD. Role of systematic reviews and meta-analysis in evidence-based clinical practice. Indian J Urol 2011; 27(4):520-524. doi: 10.4103/0970-1591.91445.

13. Clarke M. The Cochrane Collaboration and systematic reviews. Br J Surg 2007;94(4):391-2. doi: 10.1002/bjs.5812.

14. Radetti G, Kleon W, Buzi F, Crivellaro C, Pappalardo L, Di Lorgi N, Maghnie M. Thyroid function and structure are affected in childhood obesity J Clin Endocrinol Metab 2008; 93:4749-4754.

15. Vinceti M, Bonvicini F, Rothman KJ, Vescovi L, Wang F, The relation between amiotrophic lateral sclerosis and inorganic selenium in drinking water: a population based, case-control study. Environ Health 2010; 9:77. https://doi.org/10.1186/1476-069X-9-77

16. Gartner R, Gasnier BCH, Dietrich JW, Bjarne K, Angstwurm MWA Selenium supplementation in patients with autoimmune thyroiditis decreases thyroid peroxidase antibodies concentrations. J Clin Endocrinol Metab 2002; 87:1687-1691.

17. Duntas LH, Mantzou E, Koutras DA. Effects of a six-month treatment with selenomethionine in patients with autoimmune thyroiditis. Eur J Endocrinol 2003; 148:389-393

18. https://www.kezi.com/content/news/103-year-old-Spanish-Flu-survivor-getsCOVID-19-vaccine-in-Eugene--573692281.html

19. Kreft I, Stibilj V, Trkov Z. Iodine and selenium contents in pumpkin (Cucurbita pepo L.) oil and oil-cake. Eur Food Res Technol 2002; 215:279-281. https://doi.org/10.1007/s00217-002-0563-5

Editor-in-Chief

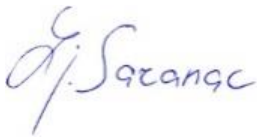

Ljiljana Šaranac 